\title{
Análisis sobre las negociaciones relativas a la deuda soberana con los holdouts argentina
}

Agustín Albini

\begin{abstract}
Resumen: Este trabajo tiene como finalidad, el estudio de la negociación que se sucedió entre Argentina y parte de los tenedores de deuda soberana argentina entre 2003 y 2016. El mismo implica, en primer lugar, la utilización de los postulados de Susan Strange, como trasfondo teórico de la cuestión, que permitan éstos un mejor entendimiento de los sucesos acontecidos. Posteriormente, realizo una contextualización de la deuda soberana argentina en años recientes, particularmente desde el año 1976 -fecha en que tomó el poder el autodenominado "Proceso de Reorganización Nacional" luego de un golpe de Estado- hasta la actualidad, que incluye un detalle de los bonos del Plan Brady ejecutado durante la década de 1990 y que son los que se encuentran en disputa. Prosigo con el estudio de las diferentes etapas del litigio con los holdouts, así como las de renegociación de deuda soberana, ya que considero a ambas indivisibles. Por último, realizo un análisis acerca de la situación final, en vistas a plantear lo que, desde mi punto de vista, representa un desafío para la deuda argentina en particular y los procesos de reestructuración de deuda en general.
\end{abstract}

Palabras clave: Deuda argentina soberana, holdouts, Griesa

\section{Analysis on the negotiations related to the argentinian sovereign debt to the holdouts}

\begin{abstract}
This article has his main objective, as to study the negotiation among Argentina and some of the Argentinian sovereign debt's holders between 2003 and 2016. It implies, at first hand, the utilization of the postulates of Susan Strange, as a theorical background of the issue, allowing a better understanding of the success that have had been place. Afterwards, I made a contextualization of the Argentinian sovereign debt in recent years, particularly from year 1976 -date where the self-called "Process of National Reorganization" assumed control after a coupe d'état- to these days, including a detail of the Brady Plan bonds, plan executed during the 1990s and which bonds are in dispute. I continue with the study of the different stages of the law suit with the holdouts, as well as the renegotiation of the sovereign debt, because I consider both indivisible. Finally, I make an analysis about the final situation, in order to suggest what, from my point of view, represents a challenge to the Argentinian debt in particular and the re-structuration debt process in general.
\end{abstract}

Keywords: Argentinian sovereign debt, holdouts, Griesa

Artigo recebido em 01/04/2016 e aprovado em 07/04/16. 


\section{Introducción}

El objetivo de este análisis, es una caracterización de la negociación existente entre el Estado argentino y parte de los tenedores de deuda soberana argentina que quedaron fuera de las dos reestructuraciones ocurridas en 2005 y 2010. Tenedores que iniciaron litigios al Estado argentino para generar el pago de las obligaciones de forma completa.

Consideramos que el abordaje de esta temática como análisis de la Política Exterior Argentina es relevante, puesto que las negociaciones de la deuda soberana han constituido un tema prioritario de la agenda externa reciente del país y una variable central en su vinculación con actores como Estados Unidos, Brasil y Venezuela, entre otros. Específicamente la cuestión de los tenedores de bonos que no ingresaron en las reestructuraciones acaecidas a lo largo de la década del kirchnerismo se han vuelto un desafío ostensible a la gestión de Cristina Fernández, tomando una serie de cursos de acción que profundizan una retórica crítica hacia los Estados Unidos, así como la propuesta de conformar y liderar el proceso de construcción de un nuevo régimen internacional relativo a la reestructuración de deudas soberanas y fundado en la experiencia argentina.

El enfoque teórico elegido sigue la línea de la teoría crítica de las Relaciones Internacionales, particularmente de las obras de Susan Strange, permitiendo generar un marco teórico para el objetivo del análisis, que diera lugar a entender mejor, desde la teoría, hechos que ocurren a diario con el sistema financiero internacional.

En primer lugar, se establecerán las formulaciones teóricas que dan sustento al análisis desplegado en este trabajo. En segundo lugar, se desarrollará una contextualización del proceso que nos condujo a estar en situación de deuda con un (actualmente) pequeño número de deudores. En este marco, se plantean formulaciones respecto al crecimiento de la deuda soberana argentina, y al desarrollo del proceso de reestructuración ocurrido entre 2001 y 2010. En relación con esto último, se dispone de una conceptualización de lo que significa un proceso de reestructuración de deuda y los riesgos que éste conlleva. Luego, se describirá el proceso existente entre el Estado argentino y los tenedores de deuda que no desearon entrar en las dos reestructuraciones, abarcando numerosas medidas legales que tomaron ambas partes. Finalmente, se realiza una conclusión sobre el tópico analizado.

\section{Los aportes de la teoría crítica para el análisis de las negociaciones en torno a la deuda soberana argentina}

Para establecer un trasfondo teórico de las negociaciones en torno a la deuda, resulta adecuado aplicar los postulados de Susan Strange] acerca del funcionamiento del sistema financiero internacional. En su libro, Dinero Loco, de 1995, la economista británica realiza un "estudio del poder en la estructura financiera durante los años ochenta y noventa"II . Según la mencionada autora, este poder está marcado por cinco grandes cambios desde mediados de la década del 80, momento en el que escribió otro de sus libros señeros: Capitalismo de casino, del año 1986.

En tal sentido, Espino González" menciona los cinco cambios: "El primero de ellos es el relacionado con los avances tecnológicos en materia de informática y telecomunicaciones [...] La segunda transformación es la referente al tamaño de los mercados financieros nacionales y globales [...] El tercer cambio es el experimentado por los intermediarios financieros bancarios [...] la cuarta transformación sustancial es 
el surgimiento y la consolidación de nuevos actores en el sistema financiero internacional",IV.

En este análisis, interesa centrarse en el cambio enunciado en último lugar, según Espino González: "el quinto y último cambio es el virtual abandono de la coordinación en la vigilancia de los intermediarios financieros por parte de los países. La autora señala que los Acuerdos de Basilea encabezados por el BIS (Banco de Acuerdos Internacionales) que promovían políticas de supervisión homólogas entre los bancos centrales miembros, han sido sustituidos por un esquema de autorregulación de los intermediarios, con consecuencias poco efectivas" $\mathrm{v}$.

En esta misma línea, Ibáñez ${ }^{\mathrm{VI}}$ sostiene que "los mecanismos e instituciones existentes para la gestión del sistema financiero siguen operando según patrones nacionales, claramente obsoletos para gestionar la integración financiera a escala mundial [...] Esta pérdida de poder no ha sido colmada por las principales organizaciones internacionales gubernamentales de carácter financiero, que han sido igualmente incapaces de controlar la evolución del sistema: el Banco de Pagos Internacionales porque se ha rendido en su lucha por adoptar y aplicar a nivel mundial estándares comunes de adecuación de capital; y el Fondo Monetario Internacional porque carece de las competencias, la pericia y los recursos necesarios para ser algo más que un prestamista de última instancia en crisis financieras como las de México en 1995 o de los países asiáticos en 1997-98" VII.

Es decir, la autora establecía la importancia de la transnacionalización de la economía, y de cómo las instituciones financieras se veían sobrepasadas e incapaces de internalizar tales cambios ya que "los estados, afirma Strange, han perdido capacidad de maniobra en su búsqueda de eficiencia, con lo que han quedado en una posición claramente desventajosa para aplicar reformas mayores al sistema"VIII. A lo que puede adicionarse que "el provecho que los operadores financieros más espabilados extrajeron de la aplicación de los cambios tecnológicos y de las innovaciones financieras, no debe hacer olvidar la connivencia (consciente e inconsciente) con la que actuaron los Estados al dejar importantes resquicios legislativos" "IX, resaltando la autora la incompetencia o inacción estatal frente a los cambios que se sucedían a nivel financiero global.

Siguiendo esta línea de Strange, en su texto, The Defective State, sostenía que los Estados detentan hoy un poder de forma colectiva, ya que de forma individual, estos han ido perdiendo atributos frente a otros actores que iban surgiendo. Esto comprende a empresas transnacionales, organizaciones intergubernamentales como el Fondo Monetario Internacional, bancos, cuentas y bufetes legales. Asimismo, el Estado comparte poder con los actores subnacionales en su interior. Expresa Strange: "La propuesta, en concreto, es que la autoridad estatal, se ha escurrido, hacia arriba, hacia abajo y hacia los costados. [...] El reinado de la anarquía en la sociedad y la economía se ha vuelto más extenso a medida que ha ido disminuyendo todo tipo de autoridad" $\mathrm{x}$. $\mathrm{Su}$ idea no implica que los Estados se han vuelto obsoletos, sino que estos se han convertido en instituciones vacías y deficientes.

A la hora de entender esta pérdida de poder, Strange comienza por analizar un punto básico de la competencia entre Estados, que es la ciencia y tecnología. La carrera que se ha generado en torno a éstas fomentó un proceso donde, además de acortarse el ciclo de vida de los bienes y servicios, se incrementó el costo para producirlos. Esto hizo que las empresas impulsaran la radicación de su producción en otros países. Desde luego, esto conllevó a una expansión de la inversión extranjera, y por tanto en una competencia de los Estados por impulsar condiciones de inversión mejores para las empresas que quisieran tener producción y/o ventas en sus territorios. Se puede ver este 


\section{AGUSTÍN ALBINI}

patrón en el hecho de que los países recientemente industrializados ofrecen mejores condiciones que los ya industrializados, o que, los países industrializados sigan apostando y promoviendo los sectores más convenientes a sus economías, fomentando la expansión de las empresas transnacionales por sobre el poder estatal, y por tanto, quitando poder de decisión al estado.

La autora también analiza la estructura actual del trabajo. Sostiene que hemos pasado de una sociedad centrada en tres factores productivos como la tierra, el trabajo y el capital hacia el capital, la información y la energía, y se ha transformado a la producción local para que sea a nivel global. Afirma Strange: “Así como estos cambios estructurales han disminuido la autoridad, la legitimidad del estado se puede resumir en 3 hipótesis [...] en primer lugar, existe un aumento en las asimetrías de la autoridad estatal, en otras palabras si el gobierno de los Estados Unidos quizás haya sufrido una pérdida de autoridad, esta pérdida refiere a los mercados y no a otros estados [...] la segunda hipótesis es que alguna autoridad sobre asuntos menos importantes políticamente han cambiado de estados locales a autoridades internacionales [...] y la tercera hipótesis es que, principalmente como resultado de la integración del mundo económico [...] hay algunas importantes responsabilidades de las autoridades políticas que ninguno en un sistema territorialmente definido de Estados está en posición de desempeñarlas"XI.

Del mismo modo, Strange expresa que como consecuencia de su intensa competencia económica, los Estados han ido mutando su accionar estatal, al punto de volverse más importante sus políticas industriales y de comercio exterior que la de Defensa. Estos cambios estructurales obligan a los Estados a procurar alianzas económicas antes que militares, tanto con otros Estados como con firmas extranjeras, dice la autora. Y este punto se relaciona con la pérdida de poder que se ha especificado más arriba: el Estado ve sobrepasada su capacidad de política exterior, al haber una gran cantidad de factores, empresas e intereses que lo presionan a la hora de las decisiones que realice.

En conclusión, la consabida idea realista de la existencia de un único centro de poder se ha ido desvaneciendo, generando así que las viejas hegemonías estatales ya no existan como tales, y se haya complejizado el estudio de las causas y consecuencias de los problemas que hoy atañen al ámbito internacional. Los procesos que hemos traído a colación y analizado, nos permiten identificar una difuminación del poder estatal a favor de actores no tradicionales, quienes desafían el accionar del Estado y limitan su capacidad de acción y reacción. //Sin embargo, esto no implica necesariamente una progresiva desaparición del Estado. Éste continúa siendo el actor principal en el sistema internacional.

\section{Una aproximación histórica: ¿Cómo llegamos a esta situación?}

La contextualización y la referencia histórica constituyen herramientas vitales para poder comprender los fenómenos que catalogamos como propios de la política reciente. En tal sentido, durante el último gobierno de facto de la Argentina autodenominado "Proceso de Reorganización Nacional" /1976 y 1983), la deuda externa creció considerablemente. Esto sucedió como consecuencia de la convergencia del interés de la banca privada de capitales en prestar el dinero que recibían por parte de los países productores de petróleo, y del gobierno argentino de obtener recursos para financiar su estrategia de reconversión de la estructura productiva de la economía argentina. Por cierto, estrategia malograda que se consustanció en proyectos ostentosos y escenarios de especulación financiera. La deuda creció, a valores nominales actuales 
de 7.901 millones de dólares en 1975 a $46.108^{\mathrm{XII}}$ millones a finales de 1983 , es decir, un $364 \%$.

El advenimiento de la denominada "década perdida" en los ochenta, marcó una etapa histórica de bajo crecimiento, alta inflación y dificultades para recomponer una economía agotada y con una alta participación estatal, que tendía a incrementar los déficits, en un contexto de bajos precios en las materias primas. Circunstancia esencial, si se tiene en cuenta que la Argentina ha dependido y depende del precio internacional de los commodities y por ende se ha beneficiado con sus momentos de gracia (como entre 2003 y 2011) y se ha perjudicado notablemente con sus ciclos de baja (como sucede en tiempos recientes).

Para 1989, luego del fracaso del Plan Baker en $1985^{\text {XIII }}$, se sucedió un período a nivel económico que devastó los pocos intentos de recuperación: la hiperinflación. Para intentar frenar esta situación, y ante el hecho de que otros planes habían fracasado, se puso en marcha la idea desarrollada por Nicholas Brady, Secretario del Tesoro de Estados Unidos, consistente en reestructurar la deuda existente, mediante la emisión de nuevos bonos. Pero para poder acceder al financiamiento externo que ofrecían estos bonos, los países debían apegarse al llamado Consenso de Washington, una serie de pautas que determinaban la desregulación de gran parte de la economía por parte del Estado, la liberalización de la misma, la privatización de un buen número de empresas estatales y la baja en el gasto estatal.

Con la llegada al gobierno de Carlos Menem (1989) y Domingo Cavallo a la cartera económica (1991), la gestión estableció la llamada "Ley de convertibilidad" en "la cual instauró la conversión de la moneda nacional a un tipo de cambio fijo de 1 peso por dólar, obligando a respaldar la base monetaria con divisas y prohibiendo cubrir el déficit fiscal a través de la emisión de moneda sin respaldo. La combinación de apertura financiera y comercial junto a la restricción monetaria en un marco de tipo de cambio bajo logró controlar la inflación, pero a costa del deterioro del entramado productivo local, profundamente afectado por la competencia externa y el incentivo a la especulación financiera"XIV.

La deuda pública negociada bajo el plan Brady tenía la particularidad de que "estar compuesta en su mayoría por títulos que cotizaban en los mercados de capital y podían ser negociados libremente por inversores individuales o institucionales (en especial fondos mutuales o de pensión)" ${ }^{\mathrm{XV}}$. Esto significó que era pasible de ser adquirida por empresas especuladoras como sucedió con NML Limited y otros de similar envergadura, actores hoy denominados holdouts, es decir aquellos que no aceptaron entrar en la reestructuración de la deuda soberana de 2005 y 2010.

De 1991 a 2001 se sucedió un período de estabilidad inflacionaria, situación inaudita en el historial económico argentino, aunque de crecimiento errático. Asimismo, la dependencia a los mercados internacionales de crédito generó una volatilidad propia de la fluctuación de capitales externos. En este contexto, se generó un círculo vicioso de endeudamiento, donde la deuda crecía, el riesgo país subía y los prestamistas se retiraban, dejando a los organismos multilaterales de crédito -el Fondo Monetario Internacional, en particular, como la única fuente de financiamento externo.

Hasta la crisis rusa de 1998 y la devaluación brasileña de 1999, Argentina sostuvo niveles de endeudamiento por debajo del $40 \%$ del PBI. Sin embargo, esta realidad se modificó raudamente luego de estos fenómenos que fomentaron la desconfianza en los inversores externos, incrementándose de modo irrefrenable la prima de riesgo exigida para la colocación de nueva deuda. Este contexto coincidió con el inicio de los vencimientos de los bonos emitidos en el marco del plan Brady. Habida 
cuenta de las contrariedades para honrarlos en forma efectiva en una coyuntura políticaeconómica adversa, las autoridades nacionales resolvieron cambiarlos por nuevos bonos con tasas más altas (ejemplo de esto son los bonos denominados FRAN, que poseen buena parte de las empresas que representan a los denominados fondos buitre).

Para el emblemático año 2001, la situación se tornó insostenible con una crisis económica y social sin precedentes, lo cual llevó a la declaración del default. Afirma Nemiña XVI: "De un stock de deuda pública a fin de 2001 de 144.500 millones de dólares, se declararon en default 94.300 millones, un 65\% del total, constituidos por 87.000 millones en títulos públicos y 7.300 millones de deuda con otros acreedores, en especial, el Club de París".

El resultado de este proceso fue la derogación de la ley de convertibilidad, la paridad con el dólar pasó a ser flotante, y los apoyos brindados por el FMI no resultaron satisfactorios o suficientes para poder palear la crisis de índole multidimensional. Sin embargo, "la estabilización del precio del dólar y la reactivación productiva por la sustitución de importaciones y las exportaciones dieron mayor certidumbre a la economía a partir de la segunda mitad de 2002"XVII. Esto sucedió a lo largo del interinato de Eduardo Duhalde, es por eso que al momento de asumir Néstor Kirchner, se encontró con la economía argentina en posición de crecimiento, impulsada por el consumo interno, un desarrollo de los sectores productivos estimulado por tipo de cambio competitivo y un aumento en el precio de las materias primas que se exportaban.

En este marco, se inició el proceso de reestructuración de deuda de la República Argentina. En este punto, resulta interesante recurrir a la conceptualización teórica de Kevin Gallagher ${ }^{\mathrm{XVIII}}$ acerca de los procesos de reestructuración de deudas soberanas: "Cuando un gobierno soberano ya no puede pagar sus deudas, las reestructuraciones de deuda soberana ocurren cuando lo que resulta es un cambio formal de contratos de deuda, negociado entre acreedores y deudores. Estas reestructuraciones o resoluciones, normalmente toman la forma de reducción de la deuda, 'cambia' bonos nuevos, con menores intereses y mayores vencimientos por los bonos en default. Tales resoluciones suelen tener altos descuentos y resultar en una pérdida para los acreedores. Estas pérdidas suelen denominarse "recortes" [...]

Existen una pluralidad de criterios porcentuales para definir cuándo una reestructuración puede ser considerada efectiva: algunos referentes de la política y la economía plantean que la misma debe ser aceptada por las dos terceras partes de los acreedores, otros marcan un piso del $75 \%$, otros del $90 \%$. Más allá del debate por los umbrales de aceptación, es de destacar que todas las posiciones parten del presupuesto de la dificultad de alcanzar el $100 \%$ de aceptación en cualquier operación de reestructuración, identificándose siempre agentes no dispuestos a aceptar los criterios de descuentos o de postergación de los vencimientos. En vistas a superar las contrariedades que supone la existencia de "'holdouts', - esto es, inversores descontentos que se niegan a negociar y reclaman el valor entero de la deuda"XIX, académicos, políticos e instituciones se dispusieron desde hace años a un debate sobre la problemática, sin que se arribara a conclusiones definitivas e institucionales al momento del estallido de la crisis de la deuda argentina ni en instancias del inicio de la reestructuración.

José Fernández Alonso nos ilustra un poco más sobre el alcance del proceso, al sostener que "lejos de presentar un análisis comparativo en detalle de los procesos de reestructuración últimos, se limitan estas líneas a señalar que «Argentina logró lo que ningún otro país había logrado antes: no sólo la quita o haircut que debieron soportar 
los tenedores de bonos alcanzó un nuevo estándar, sino que la reestructuración alcanzada logró que los acreedores aceptaran (además de la quita), una extensión en el plazo y una reducción en las tasas de interés finalmente reconocidas. Así, mientras en otras reestructuraciones los acreedores debieron aceptar algunas de las tres medidas mencionadas, en el caso argentino fueron introducidas todas. Nuevamente, y a fines ilustrativos, se advierte que mientras la quita del capital alcanzó el $66 \%$ en la reestructuración argentina, Rusia y Ecuador instrumentaron quitas del 37,5 y 40\%, respectivamente. Los mencionados procesos de canje ejecutados por Ucrania, Paquistán y Uruguay no recurrieron, huelga indicar, a esta herramienta, aunque sí a la reducción de los intereses y/o a la extensión de la madurez de deuda."XX.

\section{El conflicto entre Argentina y los holdouts}

Es posible afirmar que el conflicto pasó por un conjunto de etapas, a lo largo de las cuales lo único que no varió fue la posición de los fondos buitre. Esto es el rechazo a toda otra oferta que no fuera el pago total de la deuda.

Apenas asumió la primera magistratura, el presidente Néstor Kirchner puso en evidencia las condiciones en las que Argentina negociaría la reestructuración. En este contexto, se advierte que el presidente junto con el ministro de Economía, Roberto Lavagna, demostraron una posición confrontativa de negociación. Su primera oferta, con un $75 \%$ de quita en términos nominales, sorprendió a los organismos internacionales y a los tenedores de deuda. Sumado al hecho de que, al momento de presentarse la oferta primaria de repago de la deuda, en la Cumbre anual del FMI y el Banco Mundial en Dubai.

El Fondo Monetario fue un actor clave en las disputas financieras y por ende, en las restructuraciones de deuda soberana. Sin embargo, la posición fuertemente crítica del presidente argentino, Néstor Kirchner, respecto a los efectos perjudiciales de las medidas ortodoxas exigidas recurrentemente por el organismo y su convencimiento de los problemas que causaba en su intervención en los procesos de reestructuración, lo llevaron a la búsqueda de aislar al FMI,. En tal caso, no aceptó la opción del financiamiento, que históricamente había conllevado un camino o un paquete de medidas a adoptar a cambio del desahogo las cuales no formaban parte del plan de Kirchner de revitalizar la economía. Tampoco integraron su particular mirada del mundo y de aquel "pensar al mundo en argentino" asumido en el discurso de asunción presidencial del 25 de mayo de 2003. En consecuencia, uno de los elementos más relevantes del proceso de restructuración de la deuda soberana argentina de 2005 fue la búsqueda de prescindir de la intervención del FMI.

Ahora bien, los litigios de la República Argentina con tenedores de títulos públicos en cesación de pagos se remonta al año 2003, cuando un conjunto de fondos institucionales de alto riesgo -denominados coloquialmente como "fondos buitre"entablan demandas contra el país en el segundo juzgado del distrito de Manhattan de Estados Unidos, presidido por el juez Thomas Poole Griesa. La razón por la cual se radicaron en tal instancia judicial fue que los bonos en cesación de pagos habían sido emitidos bajo jurisdicción norteamericana, siendo por consecuencia tal tribunal el espacio natural para la resolución de disputas ligadas a dichos bonos.

Para 2003, los que tomaron el papel de fondos buitre fueron EM, de las Islas Caimán, y NML Limited, del cual aún poco se conocía ${ }^{\mathrm{XXI}}$. En el caso de EM, su titular era Kenneth Dart, y el de otrora revelado de NML Limited, Jay Newman. Su objetivo era el cobro total de la deuda que ambos tenían, resultante de los intereses y capitales impagos de los bonos FRAN, mencionados con anterioridad y como consecuencia del 
plan Brady. Estos bonos eran "instrumentos emitidos por el país a una tasa variable, calculada sobre la base del mecanismo de atar la misma al rendimiento que el mercado le asignaba a otros títulos de la Argentina". El valor nominal total de la deuda, 289 millones de dólares, al sumarse los mencionados intereses y capitales, ascendía casi 2.800 millones de dólares ${ }^{X X I I}$.

Durante la audiencia que ordenó el juez Griesa, el día 31 de octubre de 2003, el gobierno argentino y los fondos buitre sostuvieron un encuentro que marcaría el paso de las negociaciones por los años siguientes. La resolución del juez Griesa significó un apoyo al proceso de reestructuración de deuda que intentaba llevar adelante la administración de Nestor Kirchner. El magistrado sostenía entonces que: "levantar la suspensión sería acordar con los demandantes que el proceso (de reestructuración de la deuda) no está yendo a ningún lado o es malo". Asimismo, expresaba en su fallo, que implicaba una espera de 90 días para que los fondos buitre pudieran buscar activos argentinos que embargar y "no quiero tomar ninguna decisión que afecte el proceso $^{\mathrm{XXIII}}$,"

El proceso judicial transitó desde sus orígenes caminos tortuosos y difíciles de definir. Los principales obstáculos en los momentos primarios resultaron ser: el FMI que como ya se mencionó con anterioridad, fue dejado de lado-, así como la dificultad a la hora de negociar con los acreedores y los bancos de inversión y propios los fondos buitre. Desde que se presentó formalmente la oferta de la deuda en Dubai, en septiembre de 2003, el equipo de Néstor Kirchner, encabezado por Roberto Lavagna, Guillermo Nielsen y Leonardo Madcur, tuvo un trance dificultoso hasta la aceptación de la oferta. Esta oferta, se dio en términos favorables a la Argentina. El monto a reestructurar era de 81.800 millones de dólares, lo cual significaba un $42 \%$ del total. La gran cantidad de bonos viejos impidió una investigación más profunda acerca del origen o pertenencia de los mismos, y se acabó por canjearlos todos por los nuevos, que estaban distribuidos en tres tipos de bonos de acuerdo al bonista, el Par, el Discount y el Cuasi Par, nominados en diferentes monedas. También se garantizó el ingreso de los bonistas a una nueva reestructuración si esta tenía mejores condiciones. Asimismo, el valor de quita, si bien en el valor nominal fue del $75 \%$, el valor real fue de un $66 \%$. Por último, surgió el llamado Cupón PBI, un mecanismo que pagaba una ganancia adicional si el crecimiento estimado anual resultaba mayor al $5 \%$ hasta 2005, un 4,25\% hasta 2014 , y un $3 \%$ de allí en adelante ${ }^{\mathrm{XIV}}$, y que fue motivado para generar mayor confianza en los bonistas que quisieran entrar al canje ${ }^{\mathrm{XXV}}$.

En estos términos, fue lanzada la oferta en junio de 2004, pero no tuvo efecto de manera inmediata debido al accionar del magistrado norteamericano Griesa. Luego de evaluar la oferta y aprobarla, esta se hizo efectiva en junio de 2005, con una aceptación del $76 \%$ de los tenedores de deuda, una cantidad que, si bien no era muy bien vista por el FMI, significaba $2 / 3$ de los bonistas y eso era una gran cantidad, en un contexto donde la oferta era agresiva y la desconfianza hacia Argentina era alta.

Luego del cierre "exitoso" del proceso de reestructuración de 2005, se conformó una organización que nucleaba a bonistas que no habían ingresado en el canje. Es así que en 2006, se conformó la American Task Force Argentina (ATFA) una organización auspiciada por EM y NML Limited, destinada a realizar acciones de lobby en los Estados Unidos para obtener el pago de la deuda. ATFA se autodenomina como "una alianza de organizaciones unidas por un justo y equitativo reconocimiento del default argentino de 2001 y su posterior reestructuración"XXVI.

Para 2008, la ATFA decidió generar una reunión en Miami entre fondos de riesgo de inversión afectados por la cesación de pagos del país, para influir sobre 
funcionarios de Estados Unidos y asegurarse por la vía de la presión política el cobro de sus acreencias. En esta reunión se hizo presente Mariano Etchebarre, un abogado argentino con quien el ATFA compartía el panel presentado en la reunión. Etchebarre presentó un método diferente de negociación para con los acreedores que estaban allí. La propuesta de Etchebarre, conocida como reverse inquiry, consistía en que ahora eran los acreedores quienes hacían la oferta, y el gobierno, si la aceptaba, garantizarla. Esta propuesta, que tuvo un aceptable número de interesados, finalmente quedó en la nada, debido a la crisis económica que golpearía al mundo unos meses después.

Entre 2005 y 2010, buena parte de la estrategia de los fondos buitre se basó en el lobby que realizaban en Estados Unidos a favor de su causa. Su modus operandi era variado, desde insinuar a pequeños tenedores de deuda que la Argentina no quería pagar lo endeudado, y que debían reclamarlo, hasta requerir el embargo de activos argentinos para la cobra total que sostenían.

Entre 2009 y 2010 se comenzó a debatir una nueva reestructuración de la deuda, en parte para satisfacer a diversos bonistas pequeños que habían quedado afuera del canje del 2005 y que deseaban entrar. Una de las propuestas manejadas, una inyección de reservas internacionales por parte del Fondo Monetario, no prosperó. En buena parte porque Amado Boudou, ministro de Economía, no deseaba perder el grado de autonomía que la Argentina había ido ganando gradualmente frente a las políticas del Fondo. Sin embargo, la propuesta que finalmente terminó triunfando, fue la manifiesta en el Decreto de Necesidad y Urgencia (DNU) que estableció el Fondo del Bicentenario, un mecanismo legal desde el cual se podía utilizar las llamadas "reservas libres de disponibilidad"XXVII, sin que estas pudieran estar sujetas a embargos por parte de holdouts. Su aplicación se vio frenada por la negativa del entonces presidente del Banco Central, Martín Redrado, a utilizar las reservas existentes, hasta que este fue removido por decreto de su cargo por la presidenta Cristina Fernández. Asimismo, Thomas Griesa, el 12 de enero de 2010 embargó preventivamente por pedido de los fondos buitre, basándose en el principio de alter ego ${ }^{\text {XVVIII }}$, casi dos millones en una cuenta del Banco Central. Sin embargo, luego de la audiencia del 15 de enero, Griesa falló a favor de Argentina, aunque sin pronunciarse sobre el principio del alter ego.

Finalmente, Redrado renunció a su cargo, y, a fin de enfriar el asunto, la presidenta Cristina Fernández declaró derogado el decreto del Fondo del Bicentenario, para crear otros dos nuevos con el mismo objetivo, consistentes en la disposición de las reservas para el pago de la deuda, uno orientado a la pública y otro a la privada.

No obstante el apoyo brindado en las instancias previas, en abril de 2010, el juez Griesa se posicionó a favor de los fondos buitres en la teoría del alter ego, y confirmó el embargo de una cuenta en la Reserva Federal de Estados Unidos por 105 millones de dólares, decisión que el gobierno argentino apelaría. De igual modo, fue un traspié en el camino de la reestructuración, que tuvo como ofertas una quita nominal de un $66,63 \%$ que representaban 18.100 millones de dólares. La aceptación del mismo fue del $66 \%$ por parte de los acreedores. Muchos de estos eran pequeños tenedores de deuda, como por ejemplo las mutuales de maestros que Elliot decía proteger. Así, se desbarataba una de las estrategias de los fondos buitre, que era posicionarse como los protectores de los acreedores menos poderosos. En términos concretos, debe subrayarse que el segundo canje de la deuda elevó el nivel de aceptación de la reestructuración a un $92.4 \%$ de los acreedores de la deuda soberana. El restante porcentaje siguió adelante con los litigios objeto de análisis del presente trabajo.

Para julio de 2011, la Cámara de Apelaciones de Estados Unidos rechazó la posición del alter ego que sostenían los buitres sobre las reservas, con un fuerte apoyo 
estadounidense acerca de cómo el BCRA no era un instrumento gubernamental sino que cumplía sus funciones y que era normal que los bancos centrales tuvieran una fuerte interacción con los gobiernos.

Luego de que la teoría del alter ego perdiera sustento y se volviera débil, los fondos buitre optaron por otra estrategia: sostener que en las negociaciones, Argentina violó el principio de pari passu, es decir, el principio de tratamiento igualitario de los acreedores. Su estrategia se vio apoyada por el constante lobby realizado en Estados Unidos, táctica sustanciada mediante la financiación creciente de campañas políticas para lograr el apoyo de aquellos a quienes daban dinero.

En octubre de 2012, se dio uno de los casos más emblemáticos de este litigio constante, que fue el embargo de la Fragata A.R.A. Libertad en Ghana. Un juez en Accra, llamado Richard Adjei Frimpong, aceptó un pedido elevado por el fondo encabezado por el ya mencionado Paul Singer y declaró el embargo del buque escuela argentino. Para los decisores de la República Argentina, la decisión del juez de Ghana contravenía toda legislación internacional ya que la fragata no se enmarcaba dentro de la categoría de bien comercial, por tanto no era susceptible de embargo. La Argentina elevó este planteo ante el Tribunal Internacional del Mar, quien inmediatamente estableció una serie de medidas cautelares ${ }^{\mathrm{XXIX}}$. La resolución del mismo fue favorable finalmente para el país, cuando el mencionado tribunal dio por válidos los argumentos de Argentina. En función de ello, Argentina y Ghana sellaron un acuerdo en la Sede la Corte Permanente de Arbitraje en La Haya el 20 de junio de 2013, donde se tomó nota de la decisión de la Suprema Corte de Ghana de revocar el embargo contra el navío dictado por un juez de primera instancia en octubre de $2012^{\mathrm{XXX}}$.

Más allá del resultado favorable en torno al pedido de embargo de la fragata, la situación iba a continuar desmejorando para la Argentina. El 24 de octubre de 2012, el juez Thomas Griesa falló a favor de los fondos buitre y su teoría de la violación del pari passu. Lo propio hizo meses más tarde, la Corte de Apelaciones de Nueva York, quien desestimó la medida del pago efectivo ordenada primariamente por Griesa, devolviéndola por consiguiente para su revisión. Esta medida del juez de primera instancia iba a suscitar rechazo por parte del ministro de Economía del segundo período presidencial de Cristina Fernández, Hernán Lorenzino, y de la mandataria, quienes sostuvieron en reiteradas ocasiones que la Argentina no pagaría a dichos fondos. A su vez, esto originó que Griesa, programara una audiencia para el 9 de noviembre de 2012, donde ambas partes harían su descargo acerca de la situación. La audiencia resultó en el fallo conocido del 22 de noviembre de 2012, que ratificó que la Argentina debía pagar el $100 \%$ en efectivo de la deuda a los fondos buitre. Pero sucedió que, ciertas partes interesadas, los cuales eran acreedores con un gran peso y que habían entrado en la reestructuración, pidieron tomar parte del proceso. Se dice que el juez Griesa los ignoró, y que por esto la Corte de Apelaciones decidió darles lugar como "apelantes interesados no involucrados" y éstas presentaron su descargo. Entre ellos se encontraba David Martínez, dueño de Fintech, un fondo catalogado en ciertas circunstancias también de "buitreJ", quien se pronunció duramente contra Griesa.

Asimismo, personalidades destacadas y de diversos ámbitos político-ideológicos como Anne Krueger, ex Sub Gerente General del FMI, y los premios Joseph Stiglitz y Nobel Nouriel Roubini, cuestionaron el fallo del juez Griesa, respaldando consecuentemente al país.

Bajo estas circunstancias, la Corte de Apelaciones declaró en suspenso la decisión de Griesa, por un lado debido a la importancia de quienes habían apelado y por otro, por la molestia de Griesa, quien había tomado un rol más fuerte que lo 
recomendable, y estableció una audiencia para el 27 de febrero en el marco de la revisión del plan de pagos de Griesa. Momento en el cual sucedió la liberación de la Fragata Libertad y su vuelta al país el 9 de enero de 2013.

Los resultados de la audiencia del 27 de febrero de 2013 fueron que "la Corte de Apelaciones de Nueva York rechazó revisar el fallo del juez de Griesa y ahora Argentina [debía] presentar una propuesta de pago". XXXI Luego de esto, se estableció una oferta a los holdouts del mismo tenor que la de la reestructuración de 2010, la cual determinaba el pago del cupón PBI como si los fondos buitre hubieran entrado al mismo. Sin embargo, éstos rechazaron esta propuesta.

Para fines de junio, la Argentina apeló frente a la Corte Suprema de Justicia de Nueva York, basada "en argumentar que el tribunal violó la Ley de Inmunidades soberanas, al restringir el uso de activos fuera del territorio de Estados Unidos y que fue en contra de la jurisprudencia del Tribunal Supremo en relación con los remedios equitativos tradicionales. Además, los representantes argentinos afirmaron que este tipo de decisiones "pone en riesgo el proceso de reestructuración de la deuda soberana, que cuenta con el apoyo de Estados Unidos"XXXII. En septiembre de ese año Griesa reafirmó su decisión y el Estado argentino solicitó la revisión de la sentencia.

En el camino de profundizar el conflicto, y para el mes de octubre, la Corte Suprema de EEUU decidió rechazar la apelación argentina, con lo cual el país se volcó a presentar un recurso extraordinario. Los fondos, a su vez, pidieron levantar el stay de la Corte de Apelaciones, cosa que fue rechazada posteriormente. XXXIII Ya en marzo de este año, Argentina hizo efectivo el pedido a la Corte Suprema de Justicia estadounidense de que analice el caso contra los fondos buitre. La fundamentación fue que "tendría consecuencias sistémicas porque nunca más un proceso de reestructuración de deuda" podría ser confiable". XXXIV

La Corte de Apelaciones solicitó a Argentina la propuesta de pago a los buitres, la cual se estableció en canjear u\$s11.177 millones de la deuda en default, por nuevos títulos públicos por u\$s4.865 millones, monto equivalente a una quita de la deuda del $56,5 \%$. En consecuencia, la nueva deuda se descomponía en u\$s3.026 millones de Bono Descuento 2033 y u\$s1.838 millones de Global 2017. Estos nuevos bonos pagarían una renta anual de intereses de u\$s335 millones. “Además, la propuesta incluye la emisión de 6.579 millones de unidades PBI, que otorgarían un potencial máximo a cobrar de u\$s1.974 millones adicionales hasta el vencimiento, si es que la economía crece y gatilla pagos del cupón PBI en el periodo, de acuerdo con las condiciones establecidas en su emisión"XXXV.

En el mes de mayo, el Estado argentino reiteró su pedido, sosteniendo asimismo que cualquiera sea el fallo, lo acatarían. XXXVI Para junio se hizo oficial el rechazo a la apelación argentina por parte de la Corte Suprema. Asimismo, Griesa convocó a una nueva audiencia y fue levantado el stay que pesaba sobre la decisión del magistrado norteamericano, que evitaba pagar a la Argentina la deuda con los holdouts.

La Argentina solicitó reponer el stay, cosa que fue denegada por Griesa, quien confirmó otra convocatoria a las partes, en la cual bloqueó el pago que quiso hacer Argentina a sus deudores que entraron en las reestructuraciones anteriores, sosteniendo que era "ilegal". Además designó al mediador Dan Pollack, para las negociaciones para el pago de la deuda. La primera reunión entre este y una delegación argentina fue el 7 de julio. XXXVII Allí, el ministro de Economía, Axel Kicillof, especificó la postura argentina y sostuvo su posición de negociar, cuestión que los fondos buitre calificaron como inexistente. Luego hubo dos reuniones más con Pollack, con resultados infructuosos. ${ }^{\text {XXXVIII }}$ 
Durante este proceso, Argentina reafirmó la necesidad de reponer el stay, debido a que consideraba desiguales las condiciones de pago entre los holdouts y los bonistas reestructurados. Asimismo, entraron en discusión las implicancias y alcance de la cláusula de Derechos sobre Ofertas Futuras, RUFO -en inglés- si el fallo continuaba siendo adverso. Para desconcierto de ambas partes, o no, las mediaciones continuaron siendo inoperantes. El Bank of New York solicitó al juez Griesa una explicación acerca del bloqueo de su pago y como continuaba la situación, cosa que no respondió.

Las negociaciones llevadas adelante por el mediador designado por el juez Griesa, Dan Pollack, no llegaron a un desenlace acordado. Más bien, las tratativas reafirmaron la posición de los actores en la disputa prorrogando la situación de incertidumbre. De hecho, al momento del cierre del presente análisis (mediados de agosto de 2014), Argentina ha hecho manifiesta su pretensión de que Estados Unidos asuma responsabilidad en la situación, por el acontecer en su sistema judicial. En tal sentido, se publicaron en el diario Página/12 las siguientes declaraciones del canciller Héctor Timerman: "Estados Unidos debe hacerse responsable por los actos ilegales del juez Thomas Griesa", y apuntó a las interpretaciones "arbitrarias, incluso en contra de la voluntad política" del gobierno estadounidense. Del mismo modo, Timerman mencionó la cláusula pari passu, cuya interpretación "es arbitraria y se extralimita respecto de la jurisprudencia del juez" afectando a "parte de los bonistas que aceptaron el canje y no son parte del proceso"XXXIX . Estas declaraciones no hicieron más que corroborar lo que a partir de este momento fue cada vez más claro y evidenciado a través de la retórica de la presidente y de los principales ministros. Esto es, la búsqueda de colocar en el gobierno de Estados Unidos una responsabilidad por las acciones de su poder judicial en lo que interpretaba como un boicot al proceso de restructuración de la deuda soberana como un conjunto, promovido desde 2003.

\section{Conclusión: pensando a futuro}

A mediados de agosto de 2014, las disputas entre el país y los poseedores de títulos públicos declarados en cesación de pagos en 2001 se encuentra aún sin resolver. Argentina insiste en su postura de no brindarle mejores condiciones a las que ya otorgó a los deudores que ingresaron en los canjes, mientras que las entidades NML y EM Limited, en las personas de Paul Singer y Jay Newman, insisten en cobrar la totalidad de la deuda, con todos los intereses y capitales que representan. En medio, hay una gran cantidad de actores involucrados, tanto directa como indirectamente. Entre ellos, el juez Griesa, los tenedores de bonos reestructurados, el mediador Pollack y un sinnúmero de entidades y personalidades que se han pronunciado a favor y en contra de ambas partes a lo largo de esta década que ha pasado. Uno de los problemas, es que Argentina continúa perdiendo credibilidad frente a las calificadoras de riesgo, quienes desde que Argentina está en default han bajado cada vez más su calificación hacia el país.

En lo inmediato, la denominada cláusula RUFO es la contrariedad más importante, por lo que es en torno a ésta que van a seguir las negociaciones, ya que es la que establece si los reestructurados van a poder reclamar en caso de que a los buitres se les termine pagando el $100 \%$.

Por otra parte, es posible mencionar numerosos acontecimientos de importancia. En primer lugar, luego de que el juez Thomas Griesa denegara la posibilidad de pago de intereses vencidos los tenedores de bonos reestructurados bajo legislación norteamericana, ha permitido excepcionalmente en algunas ocasiones, el pago de intereses a aquellos tenedores de títulos reestructurados bajo legislación argentina. 
Asimismo y en el plano de la política doméstico argentina, el Poder Ejecutivo envió al Congreso Nacional un proyecto de ley que resultó aprobado donde efectivizó la alternativa de cambiar la jurisdicción de los bonos de Nueva York a Buenos Aires, incorporando además, que el pago puede realizarse tanto en Buenos Aires como en París. Situación que el juez Griesa calificó de "ilegal"XL.

Por otra parte, en la Organización de las Naciones Unidas (ONU), "se aprobó una resolución que establece la necesidad de generar un "marco jurídico multilateral para los procesos de reestructuración de la deuda soberana". Con respecto a esto, la presidenta Cristina Fernández sostuvo que la Argentina no está "aislada del mundo" y ha "sentado doctrina" a nivel internacional respecto de la reestructuración de deudas."XLI Asimismo, se aprobó la formación de un Consejo con el objeto de analizar si los fondos buitre han realizado violaciones de derechos humanos en esta causa. Esta búsqueda por parte de la Argentina de llevar al ámbito multilateral de las Naciones Unidas un aspecto que ha sido previamente manifestado en el ámbito del G20 como espacio de discusión de la arquitectura financiera internacional, se dio al momento de las presiones domésticas por la aprobación de la ley vinculada a la jurisdicción de los bonos. Asimismo, se desarrolló como corolario de la fuerte intención del gobierno de Fernández de culpar a los Estados Unidos por boicotear la reestructuración de la deuda por acción y por omisión.

Frente a estas acciones, el magistrado de Nueva York declaró en desacato a la Argentina, ya que considera que el gobierno está haciendo caso omiso a la sentencia del pago de la deuda que sostienen con los fondos buitre. Las declaraciones del gobierno argentino han sido que la medida es violatoria del derecho internacional, y poco importante a fines realmente prácticos.

Llegado a este punto, se podría afirmar que, los avances que ha llevado a cabo la Argentina en el plano multilateral son importantes, reafirmando la existencia de intentos por refrenar la pérdida de poder estatal frente a las corporaciones globales (retomando a Strange). Sin embargo, es dable recalcar que estos avances no son retroactivos, y que Argentina, más allá de los logros que obtenga (como el marco jurídico para los procesos de reestructuración), deberá continuar enfrentándose a su propio caso en las cortes, y con un juez que cada día descree más de la Argentina. En consecuencia y para finalizar, se reafirma la necesidad de recordar que lo que está en juego son los procesos de reestructuración de deuda, sus alcances y limitaciones, y cuán posible será llevarlos a cabo si continúan devengándose en procesos tan desgastantes para los Estados y con fuertes consecuencias sociales.

Los avances a lo largo del último año han sido leves, y la situación, en su panorama general, ha continuado sin mayores cambios. Los holdouts, ante el hecho de que Argentina no presenta ningún signo de realizar el pago que le fuera indicado hacer, por el juez de la Suprema Corte Thomas Griesa, han presentado variados recursos para obtener el dinero que creen merecer. Uno de ellos, denominado Discovery, se basa en la idea de que Argentina debe permitir el acceso, a información de cuentas bancarias en el exterior que sean plausibles de embargo. Los demandantes, aunque han logrado avances en este sentido y han podido ver cierta información, consideran que ésta en insuficiente y bogan por obtener más.

Asimismo, el juez aceptó el reclamo de una serie de deudores de bonos argentinos, que entraran en la reestructuración previa y que querían acoplarse a la demanda de NML Limited y EM, medida que se conoció como los "me too" (yo también, en inglés). Sin embargo, la Corte de Apelaciones de Nueva York, acabó por rechazar esta disposición y advertir al juez que revisara sus fallos ${ }^{\mathrm{XLII}}$. 


\section{ANÁLISIS SOBRE LAS NEGOCIACIONES RELATIVAS A LA DEUDA SOBERANA CON LOS \\ HOLDOUTS ARGENTINA. \\ AGUSTÍN ALBINI}

También, los representantes legales de los holdouts han insistido en que el nuevo bono argentino denominado BONAR 2024 es plausible de ser embargado, y el juez Griesa ha concedido audiencias para que se explayen sobre el tema. Sin embargo, entidades bancarias como el Deutsche Bank, reclaman contra algunas de las medidas llevadas adelante, como el Discovery y la obtención de información del BONAR, afirmando que "el hecho de que todos los documentos presentados hasta la fecha relativos a los bonos Bonar 2024 [y de otro tipo] no han llevado al descubrimiento de bienes embargables materiales subraya que la verdadera motivación de los demandantes no es el descubrimiento de información relevante, sino el acoso de intermediarios financieros"XLIII.

Estos son algunos de los elementos en que se concentraron los denominados fondos buitre, una vez expirada la cláusula RUFO. Las consecuencias más significativas para Argentina, implican la falta de inversión externa, un fuerte cuestionamiento de las empresas que califican los bonos ("Moody's y Standard \& Poors, dos agencias calificadoras de riesgo, expresaron que Argentina, para acceder a un mejor crédito internacional y solventar su economía, debe resolver el conflicto con los holdouts y que, entretanto no se arregle, las calificadoras mantendrán al país en una calificación de default selectivo."XLIV ), y como consecuencia, Argentina está colocando bonos (el denominado BONAR 2024) con tasas de interés que sobrepasan de forma holgada a $\operatorname{otros}^{\mathrm{XLV}}$.

Argentina y los holdouts continúan sin resolver su disputa. Esto quita credibilidad al Estado argentino, frente a otros posibles acreedores, y por tanto la situación financiera dependerá de otros medios que ya han sido utilizados, como el gasto público y la colocación de nuevos bonos. Asimismo, un panorama de exportaciones que se muestra nebuloso, frente a la recesión y devaluación brasileña, a la desaceleración china y a la baja en los precios de materias primas, tampoco brinda seguridad ni las divisas suficientes para hacer frente al gasto público que se sostiene. Si Argentina quiere conseguir mejor crédito a nivel internacional, uno o más de estos factores deberá cambiar de forma positiva.

\section{Notas}

' Quiero agradecer dos académicos que dieron su apoyo a la elaboración de este trabajo. En primer lugar, al Doctor José Fernández Alonso, quien me ayudó constantemente en el desarrollo de este documento, y a la Doctora María del Pilar Bueno Rubial, debido a que sin su impulso y apoyo esto no hubiera sido posible.

II IBAÑEZ, Josep (2000), Reseña del libro "Dinero Loco. El descontrol del sistema financiero global", en Revista Electrónica de Estudios Internacionales, $N^{\circ}$ 1, diciembre, disponible en www.reei.org

III ESPINO GONZÁLEZ, Luis Antonio (1999), "Susan Strange, Mad Money: When Money outgrow governments", Centro de Investigación y Docencia Económicas, disponible en http://aleph.academica.mx/jspui/handle/56789/11603, último acceso agosto de 2014.

Iv ESPINO GONZÁLEZ, Luis Antonio (1999), "Susan Strange, Mad Money: When Money outgrow governments", Centro de Investigación y Docencia Económicas, disponible en http://aleph.academica.mx/jspui/handle/56789/11603, último acceso agosto de 2014.

v ESPINO GONZÁLEZ, Luis Antonio (1999), "Susan Strange, Mad Money: When Money outgrow governments", Centro de Investigación y Docencia Económicas, disponible en http://aleph.academica.mx/jspui/handle/56789/11603, último acceso agosto de 2014.

VI IBAÑEZ, Josep (2000), Reseña del libro "Dinero Loco. El descontrol del sistema financiero global", en Revista Electrónica de Estudios Internacionales, $\mathrm{N}^{\circ} 1$, diciembre, disponible en www.reei.org

VII IBAÑEZ, Josep (2000), Reseña del libro "Dinero Loco. El descontrol del sistema financiero global", en Revista Electrónica de Estudios Internacionales, $\mathrm{N}^{\circ}$ 1, diciembre, disponible en www.reei.org 


\section{ANÁLISIS SOBRE LAS NEGOCIACIONES RELATIVAS A LA DEUDA SOBERANA CON LOS \\ HOLDOUTS ARGENTINA. \\ AGUSTÍN ALBINI}

VIII ESPINO GONZÁLEZ, Luis Antonio (1999), "Susan Strange, Mad Money: When Money outgrow governments", Centro de Investigación y Docencia Económicas, disponible en http://aleph.academica.mx/jspui/handle/56789/11603, último acceso agosto de 2014.

IX IBAÑEZ, Josep (2000), Reseña del libro "Dinero Loco. El descontrol del sistema financiero global”, en Revista Electrónica de Estudios Internacionales, $\mathrm{N}^{\circ} 1$, diciembre, disponible en www.reei.org

x STRANGE, Susan (1995) "The Defective State" disponible en http://www.jstor.org/stable/20027297, último acceso octubre de 2014.

XI STRANGE, Susan (1995) "The Defective State" disponible en http://www.jstor.org/stable/20027297, último acceso octubre de 2014.

XII Ampliar en World Data Bank, disponible en http://databank.worldbank.org/, consultado en agosto de 2014.

XIII El Plan Baker fue una iniciativa de James Baker, por entonces Secretario del Tesoro de Estados Unidos. La misma consistió en una serie de políticas de ajuste y apertura por parte de los países latinoamericanos, a fin de lograr una mayor inversión y ahorro, impulsadas por un aporte financiero de 47.000 millones de dólares. Información disponible en http://www.argentina-rree.com/11/11-076.htm, consultado en agosto de 2014. El Plan Baker suponía que la crisis era un problema de liquidez y no de solvencia, como más tarde se planteó.

xIV NEMIÑA, Pablo (2012), “Argentina: camino del desendeudamiento (1991-2011)”, en Ola Financiera, UNAM, Año 5, N¹2, pp. 70-88.

xv NEMIÑA, Pablo (2012), “Argentina: camino del desendeudamiento (1991-2011)”, en Ola Financiera, UNAM, Año 5, Nº12, pp. 70-88.

XVI NEMIÑA, Pablo (2012), “Argentina: camino del desendeudamiento (1991-2011)”, en Ola Financiera, UNAM, Año 5, N¹2, pp. 70-88.

XVII NEMIÑA, Pablo (2012), “Argentina: camino del desendeudamiento (1991-2011)”, en Ola Financiera, UNAM, Año 5, N¹2, pp. 70-88.

xVIII GALLAGHER, Kevin (2011), "The New Vulture Culture: Sovereign debt restructuring and trade and investment treaties", The ideas working papers series, $\mathrm{N}^{\circ} 2$, disponible en http://www.ase.tufts.edu/gdae/publications/GallagherSovereignDebt.pdf, último acceso agosto de 2014.

XIX GALLAGHER, Kevin (2011), "The New Vulture Culture: Sovereign debt restructuring and trade and investment treaties", The ideas working papers series, $\mathrm{N}^{\circ} 2$, disponible en http://www.ase.tufts.edu/gdae/publications/GallagherSovereignDebt.pdf, último acceso agosto de 2014.

xx FERNANDEZ ALONSO, José Marcelino (2010), "La cuestión de la reinserción financiera en la política exterior de la República Argentina tras el proceso de reestructuración de la deuda (2005-2009)" en BOLOGNA, Bruno "La política exterior del gobierno de Cristina Fernández: apreciaciones promediando su mandato", $\quad \mathrm{su}$ disponible http://www.cerir.com.ar/admin/_cerir/archivos/libros/0000136/Tomo\%20V\%20-\%20tapa\%20color.pdf

${ }^{\mathrm{XXI}}$ LAUDONIA, Mara (2013), "Los Buitres de la Deuda”, Editorial Biblos, Buenos Aires.

xxII LAUDONIA, Mara (2013), "Los Buitres de la Deuda", Editorial Biblos, Buenos Aires.

XXIII Diario Página 12, disponible en http://www.pagina12.com.ar/diario/economia/2-27583-2003-1101.html, consultado en agosto de 2014.

XXIV Ampliar en Página 12, disponible en http://www.pagina12.com.ar/diario/economia/2-46086-200501-13.html, consultado en agosto de 2014 y en Fernández Alonso, 2006.

xxv LAUDONIA, Mara (2013), "Los Buitres de la Deuda", Editorial Biblos, Buenos Aires.

XXVI Para ampliar ver en http://www.atfa.org/about-us/, consultada en agosto de 2014.

XXVII Las reservas de libre disponibilidad son aquellas que se cuentan luego de haber completado la cantidad necesaria de dinero para respaldar la base monetaria existente. Es decir, si las reservas significan el $120 \%$ de la base monetaria, el $20 \%$ excedente son reservas de libre disponibilidad.

XXVIII El principio de alter ego, implicaba, según la demanda de EM Limited y NML Limited, que el Banco Central de la República Argentina no funcionaba como un órgano independiente si no que era una extensión del poder Ejecutivo.

XXIX LAUDONIA, Mara (2013), "Los Buitres de la Deuda”, Editorial Biblos, Buenos Aires.

XXX Informe OPEA 278, disponible en www.opeargentina.org consultado en agosto de 2014.

XXXI Informe OPEA 251, disponible en www.opeargentina.org, consultado en agosto de 2014.

XXXII Informe OPEA 264, disponible en www.opeargentina.org, consultado en agosto de 2014.

xxxIII Informes OPEA 280 y 283, disponibles en www.opeargentina.org, consultado en agosto de 2014. 


\section{ANÁLISIS SOBRE LAS NEGOCIACIONES RELATIVAS A LA DEUDA SOBERANA CON LOS \\ HOLDOUTS ARGENTINA. \\ AGUSTÍN ALBINI}

XXXIV Informes OPEA 280 y 283, disponibles en www.opeargentina.org, consultado en agosto de 2014. XXXV BRODERSOHN, Mario; CASTIÑEIRA, Ramiro (2013), "Oferta oficial a los holdouts: replicar el canje 2010", Informe mensual Econométrica, marzo, disponible en http://www.econometrica.com.ar/productosservicios/archivos/mensual/Econometrica\%20Quantum\%20\%20Informe\%20Mensual\%20-\%20Marzo\%202013.pdf, último acceso en agosto de 2014. XXXVI Informe OPEA 305, disponible en www.opeargentina.org, consultado en agosto de 2014. XXXVII Informe OPEA 310, disponible en www.opeargentina.org, consultado en agosto de 2014. XXXVIII Informe OPEA 311, disponible en www.opeargentina.org, consultado en agosto de 2014. XxxIX Página 12, disponible en http://www.pagina12.com.ar/diario/ultimas/20-253311-2014-08-19.html, consultado en agosto de 2014.

${ }^{\mathrm{XL}}$ Informe OPEA 318, disponible en www.opeargentina.org, consultado en octubre de 2014.

XLI Informe OPEA 320, disponible en www.opeargentina.org, consultado en octubre de 2014.

XLII Informe OPEA 358, disponible en www.opeargentina.org, consultado en agosto de 2015.

XLIII Informe OPEA 353, disponible en www.opeargentina.org, consultado en agosto de 2015.

XLIV Informe OPEA 359, disponible en www.opeargentina.org, consultado en agosto de 2015.

${ }^{X L V}$ El BONAR ofrece tasas del 8,75\% anual a 10 años, mientras que un bono promedio estadounidense en el mismo período, ofrece entre un 2 y $2,2 \%$.

\section{BIBLIOGRAFÍA}

BRODERSOHN, Mario; CASTIÑEIRA, Ramiro (2013), "Oferta oficial a los holdouts: replicar el canje 2010”, Informe mensual Econométrica, marzo, disponible en http://www.econometrica.com.ar/productosservicios/archivos/mensual/Econometrica\%2 0Quantum\%20-\%20Informe\%20Mensual\%20-\%20Marzo\%202013.pdf, último acceso en agosto de 2014.

COOPER, Andrew y MOMANI, Bessma (2005), 'Negotiating Out of Argentina's Financial Crisis: Segmenting the International Creditors, en New Political Economy, Vol. 10, No. 3, September 2005, disponible en http://www.arts.uwaterloo.ca/ bmomani/Documents/NPE_Negotiating_Out_of_Argenti na.pdf

ESCUDÉ, Carlos, CISNEROS, Andrés (2000), Historia de las Relaciones Exteriores Argentina, disponible en http://www.argentina-rree.com/11/11-076.htm, último acceso en agosto de 2014.

ESPINO GONZÁLEZ, Luis Antonio (1999), "Susan Strange, Mad Money: When Money outgrow governments", Centro de Investigación y Docencia Económicas, disponible en http://aleph.academica.mx/jspui/handle/56789/11603, último acceso agosto de 2014.

FERNANDEZ ALONSO, José Marcelino (2010), "La cuestión de la reinserción financiera en la política exterior de la República Argentina tras el proceso de reestructuración de la deuda (2005-2009)" en BOLOGNA, Bruno "La política exterior del gobierno de Cristina Fernández: apreciaciones promediando su mandato", disponible 
http://www.cerir.com.ar/admin/_cerir/archivos/libros/0000136/Tomo\%20V\%20\%20tapa\%20color.pdf

GALLAGHER, Kevin (2011), "The New Vulture Culture: Sovereign debt restructuring and trade and investment treaties”, The ideas working papers series, $\mathrm{N}^{\circ} 2$, disponible en http://www.ase.tufts.edu/gdae/publications/GallagherSovereignDebt.pdf, último acceso agosto de 2014.

IBAÑEZ, Josep (2000), Reseña del libro "Dinero Loco. El descontrol del sistema financiero global", en Revista Electrónica de Estudios Internacionales, $\mathrm{N}^{\circ} 1$, diciembre, disponible en www.reei.org

INFORMES del OBSERVATORIO DE POLÍTICA EXTERIOR ARGENTINA, disponibles en www.opeargentina.org/, último acceso en agosto de 2015.

LAUDONIA, Mara (2013), "Los Buitres de la Deuda", Editorial Biblos, Buenos Aires.

NEMIÑA, Pablo (2012), “Argentina: camino del desendeudamiento (1991-2011)", en Ola Financiera, UNAM, Año 5, N${ }^{\circ} 12$, pp. 70-88.

STRANGE, Susan (1995) "The Defective State" disponible en http://www.jstor.org/stable/20027297, último acceso octubre de 2014.

STURZENEGGER, Federico y ZETTELMEYER, Jeromin (2006) "Debt, Defaults and Lessons from a Decade of Crises", Massachusetts Institute of Technology.

\section{Sitios web consultados}

American Task Force Argentina en http://www.atfa.org

Diario Página 12 en http://www.pagina12.com.ar

World Data Bank en http://databank.worldbank.org/ 